\title{
Zeros of the Derivatives of $L$-Functions Attached to Cusp Forms
}

by

\author{
Yoshikatsu YASHIRO \\ Presented by Jerzy KACZOROWSKI
}

Summary. Let $f$ be a holomorphic cusp form of weight $k$ with respect to $\mathrm{SL}_{2}(\mathbb{Z})$ which is a normalized Hecke eigenform, and $L_{f}(s)$ the $L$-function attached to $f$. We shall give a relation between the number of zeros of $L_{f}(s)$ and of the derivatives of $L_{f}(s)$ using Berndt's method, and an estimate of zero-density of the derivatives of $L_{f}(s)$ based on Littlewood's method.

1. Introduction. Let $f$ be a cusp form of weight $k$ for $\mathrm{SL}_{2}(\mathbb{Z})$ which is a normalized Hecke eigenform. Let $a_{f}(n)$ be the $n$th Fourier coefficient of $f$ and set $\lambda_{f}(n)=a_{f}(n) / n^{(k-1) / 2}$. Rankin showed that $\sum_{n \leq x}\left|\lambda_{f}(n)\right|^{2}=$ $C_{f} x+O\left(x^{3 / 5}\right)$ for $x \in \mathbb{R}_{>0}$, where $C_{f}$ is a positive constant depending on $f$ (see [8, (4.2.3), p. 364]). The $L$-function attached to $f$ is defined by

$$
L_{f}(s)=\sum_{n=1}^{\infty} \frac{\lambda_{f}(n)}{n^{s}}=\prod_{p \text { prime }}\left(1-\frac{\alpha_{f}(p)}{p^{s}}\right)^{-1}\left(1-\frac{\beta_{f}(p)}{p^{s}}\right)^{-1} \quad(\operatorname{Re} s>1),
$$

where $\alpha_{f}(p)$ and $\beta_{f}(p)$ satisfy $\alpha_{f}(p)+\beta_{f}(p)=\lambda_{f}(p)$ and $\alpha_{f}(p) \beta_{f}(p)=1$. By Hecke's work [4], the function $L_{f}(s)$ is analytically continued to the whole $s$-plane by

$$
(2 \pi)^{-s-(k-1) / 2} \Gamma\left(s+\frac{k-1}{2}\right) L_{f}(s)=\int_{0}^{\infty} f(i y) y^{s+(k-1) / 2-1} d y,
$$

2010 Mathematics Subject Classification: Primary 11M26; Secondary 11N75.

Key words and phrases: cusp forms, $L$-functions, derivatives, zeros.

Received 24 May 2016.

Published online 27 October 2016. 
and has a functional equation

$$
L_{f}(s)=\chi_{f}(s) L_{f}(1-s)
$$

where $\chi_{f}(s)$ is given by

$$
\begin{aligned}
\chi_{f}(s) & =(-1)^{-k / 2}(2 \pi)^{2 s-1} \frac{\Gamma\left(1-s+\frac{k-1}{2}\right)}{\Gamma\left(s+\frac{k-1}{2}\right)} \\
& =2(2 \pi)^{-2(1-s)} \Gamma\left(s+\frac{k-1}{2}\right) \Gamma\left(s-\frac{k-1}{2}\right) \cos (\pi(1-s)) .
\end{aligned}
$$

The second equality is deduced from the fact that $\Gamma(s) \Gamma(1-s)=\pi / \sin (\pi s)$ and $\sin (\pi(s+(k-1) / 2))=(-1)^{k / 2} \cos (\pi(1-s))$. Similarly to the case of the Riemann zeta function $\zeta(s)$, it is conjectured that all complex zeros of $L_{f}(s)$ lie on the critical line $\operatorname{Re} s=1 / 2$, which is the Generalized Riemann Hypothesis (GRH). In order to support the truth of the GRH, the distribution and the density of complex zeros of $L_{f}(s)$ are studied without assuming the GRH.

Lekkerkerker [6] proved an approximate formula for the number of complex zeros of $L_{f}(s)$ :

$$
N_{f}(T)=\frac{T}{\pi} \log \frac{T}{2 \pi e}+O(\log T),
$$

where $T>0$ is sufficiently large, and $N_{f}(T)$ denotes the number of complex zeros of $L_{f}(s)$ in $0<\operatorname{Im} s \leq T$. The formula (1.4) is analogous to the formula for $N(T)$, the number of complex zeros of $\zeta(s)$ in $0<\operatorname{Im} s \leq T$, given by Riemann [9]:

$$
N(T)=\frac{T}{2 \pi} \log \frac{T}{2 \pi e}+O(\log T) .
$$

(Later von Mangoldt [14] proved (1.5) rigorously.) In the Riemann zeta function, the zeros of the derivative of $\zeta(s)$ have a connection with the Riemann Hypothesis (RH). Speiser [10] showed that RH is equivalent to the non-existence of a complex zero of $\zeta^{\prime}(s)$ in $\operatorname{Re} s<1 / 2$. Levinson and Montgomery [7] proved that if $\mathrm{RH}$ is true, then $\zeta^{(m)}(s)$ has at most finitely many complex zeros in $0<\operatorname{Re} s<1 / 2$ for any $m \in \mathbb{Z}_{\geq 0}$.

There are many studies of the zeros of $\zeta^{(m)}(s)$ without assuming RH. Spira [11], [12] showed that there exist $\sigma_{m} \geq(7 m+8) / 4$ and $\alpha_{m}<0$ such that $\zeta^{(m)}(s)$ has no zero with $\operatorname{Re} s \leq \sigma_{m}$ or $\operatorname{Re} s \leq \alpha_{m}$, and exactly one real zero in each open interval $(-1-2 n, 1-2 n)$ for $1-2 n \leq \alpha_{m}$. Later, Yildirım [16] showed that $\zeta^{\prime \prime}(s)$ and $\zeta^{\prime \prime \prime}(s)$ have no zeros in $0 \leq \operatorname{Re} s<1 / 2$. Berndt [2] gave a relation between the numbers of complex zeros of $\zeta(s)$ and of $\zeta^{(m)}(s)$ :

$$
N_{m}(T)=N(T)-\frac{T \log 2}{2 \pi}+O(\log T)
$$


where $m \in \mathbb{Z}_{\geq 1}$ is fixed and $N_{m}(T)$ denotes the number of complex zeros of $\zeta^{(m)}(s)$ in $0<\operatorname{Im} s \leq T$. Recently, Aoki and Minamide [1] studied the density of zeros of $\zeta^{(m)}(s)$ in the right hand side of the critical line $\operatorname{Re} s=$ $1 / 2$ by using Littlewood's method. Let $N_{m}(\sigma, T)$ be the number of zeros of $\zeta^{(m)}(s)$ in $\operatorname{Re} s \geq \sigma$ and $0<\operatorname{Im} s \leq T$. They showed that

$$
N_{m}(\sigma, T)=O\left(\frac{T}{\sigma-1 / 2} \log \frac{1}{\sigma-1 / 2}\right),
$$

uniformly for $\sigma>1 / 2$. From $(1.6)$ and $(1.7)$, we see that almost all complex zeros of $\zeta^{(m)}(s)$ lie in the neighbourhood of the critical line.

The purpose of this paper is to study the counterparts of the results of Berndt, Aoki and Minamide for the derivatives of $L_{f}(s)$, namely, the relation between the number of complex zeros of $L_{f}(s)$ and that of $L_{f}^{(m)}(s)$, and the density of zeros of $L_{f}^{(m)}(s)$ in the right half-plane Re $s>1 / 2$. Let $n_{f}$ be the smallest integer greater than 1 such that $\lambda_{f}\left(n_{f}\right) \neq 0$. The $m$ th derivative of $L_{f}(s)$ is given by

$$
L_{f}^{(m)}(s)=\sum_{n=1}^{\infty} \frac{\lambda_{f}(n)(-\log n)^{m}}{n^{s}}=\sum_{n=n_{f}}^{\infty} \frac{\lambda_{f}(n)(-\log n)^{m}}{n^{s}} \quad(\operatorname{Re} s>1),
$$

Differentiating both sides of 1.2 , we find that $L_{f}^{(m)}(s)$ is holomorphic in the whole $s$-plane and has the functional equation

$$
L_{f}^{(m)}(s)=\sum_{r=0}^{m}\left(\begin{array}{c}
m \\
r
\end{array}\right)(-1)^{r} \chi_{f}^{(m-r)}(s) L_{f}^{(r)}(1-s)
$$

First, we shall exhibit zero free regions for $L_{f}^{(m)}(s)$ by following Berndt's method (see [2]) and Spira's method (see [11], [12]).

THEOREM 1.1. The following assertions hold for any $m \in \mathbb{Z}_{\geq 0}$.

(i) There exists $\sigma_{f, m} \in \mathbb{R}_{>1}$ such that $L_{f}^{(m)}(s)$ has no zero in $\operatorname{Re} s \geq$ $\sigma_{f, m}$.

(ii) For any $\varepsilon \in \mathbb{R}_{>0}$, there exists $\delta_{f, m, \varepsilon} \in \mathbb{R}_{>(k-1) / 2+1}$ such that $L_{f}^{(m)}(s)$ has no zero with $|s| \geq \delta_{f, m, \varepsilon}$ satisfying $\operatorname{Re} s \leq-\varepsilon$ and $|\operatorname{Im} s| \geq \varepsilon$.

(iii) There exists $\alpha_{f, m} \in \mathbb{R}_{<-(k-1) / 2-1}$ such that $L_{f}^{(m)}(s)$ has only real zeros in $\operatorname{Re} s \leq \alpha_{f, m}$, and one real zero in each interval $(n-1, n)$ for $n \in \mathbb{Z}_{\leq \alpha_{f, m}}$.

Next, based on Berndt's proof, we can obtain the following formula for the number of complex zeros of $L_{f}^{(m)}(s)$ :

TheOREM 1.2. For any fixed $m \in \mathbb{Z}_{\geq 1}$, let $N_{f, m}(T)$ be the number of complex zeros of $L_{f}^{(m)}(s)$ in $0<\operatorname{Im} s \leq T$. Then for any large $T>0$, we 
have

$$
N_{f, m}(T)=\frac{T}{\pi} \log \frac{T}{2 \pi e}-\frac{T}{2 \pi} \log n_{f}+O(\log T) .
$$

Moreover the relation between $N_{f}(T)$ and $N_{f, m}(T)$ is given by

$$
N_{f, m}(T)=N_{f}(T)-\frac{T}{2 \pi} \log n_{f}+O(\log T) .
$$

Finally, using the mean value formula for $L_{f}^{(m)}(s)$ obtained in [15] and Littlewood's method, we obtain the estimate of the density of zeros:

Theorem 1.3. For any $m \in \mathbb{Z}_{\geq 0}$, let $N_{f, m}(\sigma, T)$ be the number of complex zeros of $L_{f}^{(m)}(s)$ with $\operatorname{Re} s \geq \sigma$ and $0<\operatorname{Im} s \leq T$. For any large $T>0$, we have

$$
N_{f, m}(\sigma, T)=O\left(\frac{T}{\sigma-1 / 2} \log \frac{1}{\sigma-1 / 2}\right)
$$

uniformly for $1 / 2<\sigma \leq 1$. More precisely,

$$
N_{f, m}(\sigma, T)
$$

$$
\begin{aligned}
\leq & \frac{2 m+1}{2 \pi} \frac{T}{\sigma-1 / 2} \log \frac{1}{\sigma-1 / 2}+\frac{1}{2 \pi} \frac{T}{\sigma-1 / 2} \log \frac{(2 m) ! n_{f} C_{f}}{\left|\lambda_{f}\left(n_{f}\right)\right|^{2}\left(\log n_{f}\right)^{2 m}} \\
& +O\left(\frac{\log T}{\sigma-1 / 2}\right)
\end{aligned}
$$

$$
+\frac{1}{2 \pi} \frac{T}{\sigma-1 / 2} \begin{cases}\log \left(1+O\left(\frac{(2 \sigma-1)^{2 m+1}(\log T)^{2 m}}{T^{2 \sigma-1}}\right)\right), & 1 / 2<\sigma<1, \\ \log \left(1+O\left(\frac{(2 \sigma-1)^{2 m+1}(\log T)^{2 m+2}}{T}\right)\right), & \sigma=1, \\ \log \left(1+O\left(\frac{(2 \sigma-1)^{2 m+1}}{T}\right)\right), & 1<\sigma<\sigma_{f, m},\end{cases}
$$

where $\sigma_{f, m}$ is given by Theorem 1.1(i).

2. Proof of Theorem 1.1. To show (i), we write

$$
L_{f}^{(m)}(s)=\lambda_{f}\left(n_{f}\right)\left(-\log n_{f}\right)^{m} F(s) n_{f}^{-s}
$$

where

$$
F(s)=1+\sum_{n=n_{f}+1}^{\infty} \frac{\lambda_{f}(n)}{\lambda_{f}\left(n_{f}\right)}\left(\frac{\log n}{\log n_{f}}\right)^{m}\left(\frac{n_{f}}{n}\right)^{s} \quad(\operatorname{Re} s>1)
$$


Deligne's result $\left|\lambda_{f}(n)\right| \leq d(n) \ll n^{\varepsilon}$ implies that there exist $c_{f} \in \mathbb{R}_{>0}$ and $\sigma_{f, m} \in \mathbb{R}_{>1}$ depending on $f$ and $m$ such that

$$
\begin{aligned}
|F(\sigma+i t)-1| & \leq \sum_{n=n_{f}+1}^{\infty}\left|\frac{\lambda_{f}(n)}{\lambda_{f}\left(n_{f}\right)}\right|\left(\frac{\log n}{\log n_{f}}\right)^{m}\left(\frac{n_{f}}{n}\right)^{\sigma} \\
& \leq c_{f} \sum_{n=n_{f}+1}^{\infty} \frac{\left(\log n / \log n_{f}\right)^{m}}{\left(n / n_{f}\right)^{\sigma-\varepsilon}} \leq \frac{1}{2}
\end{aligned}
$$

for $\sigma \in \mathbb{R}_{\geq \sigma_{f, m}}$ and $t \in \mathbb{R}$, where $\varepsilon$ is an arbitrary positive number. Hence $L_{f}^{(m)}(s)$ has no zeros with $\operatorname{Re} s \geq \sigma_{f, m}$, that is, (i) is proved.

Next we shall show (iii) and (iii). Replacing $s$ with $1-s$ in (1.3) and (1.8), we have

$$
\begin{aligned}
& \quad(-1)^{m} L_{f}^{(m)}(1-s) \\
& =\sum_{r=0}^{m}\left(\begin{array}{c}
m \\
r
\end{array}\right) L_{f}^{(m-r)}(s)\left(2(2 \pi)^{-2 s} \cos (\pi s) \Gamma\left(s-\frac{k-1}{2}\right) \Gamma\left(s+\frac{k-1}{2}\right)\right)^{(r)} .
\end{aligned}
$$

Since $(\cos (\pi s))^{(r)}=\pi^{r}\left(a_{r} \cos (\pi s)+b_{r} \sin (\pi s)\right)$ where $a_{r}, b_{r} \in\{0, \pm 1\}$, and $\left((2 \pi)^{-2 s}\right)^{(r)}=(-2 \log 2 \pi)^{r}(2 \pi)^{-2 s}$ for $r \in \mathbb{Z}_{\geq 0}$, the formula 2.3) can be written as

$$
\begin{aligned}
& (-1)^{m} L_{f}^{(m)}(1-s) \\
& \quad=2(2 \pi)^{-2 s} \sum_{r=0}^{m} R_{m-r}(s)\left(\Gamma\left(s-\frac{k-1}{2}\right) \Gamma\left(s+\frac{k-1}{2}\right)\right)^{(r)}
\end{aligned}
$$

where

$$
R_{m-r}(s)=\cos (\pi s) \sum_{j=0}^{m-r} a_{j}^{\prime} L_{f}^{(j)}(s)+\sin (\pi s) \sum_{j=0}^{m-r} b_{j}^{\prime} L_{f}^{(j)}(s)
$$

and $a_{r}^{\prime}, b_{r}^{\prime} \in \mathbb{R}$. It is clear that $a_{0}^{\prime}=1, b_{0}^{\prime}=0$ and $R_{0}(s)=L_{f}(s) \cos (\pi s)$. Moreover we write (2.4) as

$$
\frac{(-1)^{m} L_{f}^{(m)}(1-s)}{2(2 \pi)^{-2 s}}=f(s)+g(s)
$$

where

$$
\begin{aligned}
& f(s)=R_{0}(s)\left(\Gamma\left(s-\frac{k-1}{2}\right) \Gamma\left(s+\frac{k-1}{2}\right)\right)^{(m)}, \\
& g(s)=\sum_{r=0}^{m-1} R_{m-r}(s)\left(\Gamma\left(s-\frac{k-1}{2}\right) \Gamma\left(s+\frac{k-1}{2}\right)\right)^{(r)} .
\end{aligned}
$$


The formula 2.5) implies that if $|f(s)|>|g(s)|$ in some region then $L_{f}^{(m)}(s)$ has no zero in that region.

In order to investigate the behavior of $f(s)$ and $g(s)$, we shall consider the approximate formula for $\left(\Gamma\left(s-\frac{k-1}{2}\right) \Gamma\left(s+\frac{k-1}{2}\right)\right)^{(r)} /\left(\Gamma\left(s-\frac{k-1}{2}\right) \Gamma\left(s+\frac{k-1}{2}\right)\right)$. By Stirling's formula, it is known that

$$
\frac{\Gamma^{\prime}}{\Gamma}(s)=\log s-\frac{1}{2 s}+\int_{0}^{\infty} \frac{\{u\}-1 / 2}{(u+s)^{2}} d u
$$

for $s \in \mathbb{C}$ such that $|\arg s| \leq \pi-\delta$ where $\delta \in \mathbb{R}_{>0}$ is fixed (see [5, Theorem A.5 b)]). Let $D:=\mathbb{C} \backslash\{s \in \mathbb{C}|\operatorname{Re} s<\varepsilon,| \operatorname{Im} s \mid<\varepsilon\}$, where $\varepsilon$ is any fixed positive number. Setting $G^{(j)}(s):=\left(d^{j-1} / d s^{j-1}\right) G^{(1)}(s)$ for $j \in \mathbb{Z}_{\geq 1}$ and $s \in D$ where $G^{(1)}(s)$ is the right-hand side of $(2.6)$, we shall use the following lemma:

Lemma 2.1 ([15, Lemma 2.3]). Let $F$ and $G$ be holomorphic functions such that $F(s) \neq 0$ and $\log F(s)=G(s)$ for $s \in D$, where $D \subset \mathbb{C}$ is a domain. Then for any fixed $r \in \mathbb{Z}_{\geq 1}$, there exist $l_{1}, \ldots, l_{r} \in \mathbb{Z}_{\geq 0}$ and $C_{\left(l_{1}, \ldots, l_{r}\right)} \in \mathbb{Z}_{\geq 0}$ such that

$$
\frac{F^{(r)}}{F}(s)=\sum_{1 l_{1}+\cdots+r l_{r}=r} C_{\left(l_{1}, \ldots, l_{r}\right)}\left(G^{(1)}(s)\right)^{l_{1}} \cdots\left(G^{(r)}(s)\right)^{l_{r}}
$$

for $s \in D$. In particular, $C_{(r, 0, \ldots, 0)}=1$.

The estimates

$$
\begin{aligned}
|u+s|^{2} & =u^{2}+|s|^{2}+2 u|s| \cos \arg s \\
& \geq \begin{cases}|s|^{2}, & u \leq|s|,|\arg s| \leq \pi / 2, \\
|s|^{2}(\sin \arg s)^{2}, & u \leq|s|, \pi / 2 \leq|\arg s| \leq \pi-\delta, \\
u^{2}, & u \geq|s|,|\arg s| \leq \pi / 2, \\
4(1+\cos \arg s) u^{2}, & u \geq|s|, \pi / 2 \leq|\arg s| \leq \pi-\delta\end{cases}
\end{aligned}
$$

give

$$
\int_{0}^{\infty} \frac{\{u\}-1 / 2}{(u+s)^{j+1}} d u \ll \int_{0}^{|s|} \frac{d u}{|s|^{j+1}}+\int_{|s|}^{\infty} \frac{d u}{u^{j+1}} \ll \frac{1}{|s|^{j}}
$$

for $j \in \mathbb{Z}_{\geq 1}$ and $s \in D$. Then

$$
\begin{aligned}
G^{(1)}(s) & =\log s+O\left(\frac{1}{|s|}\right), \\
G^{(j)}(s) & =\frac{(-1)^{j-1}(j-2) !}{s^{j-1}}+\frac{(-1)^{j}(j-1) !}{2 s^{j}}+(-1)^{j+1} j ! \int_{0}^{\infty} \frac{\{u\}-1 / 2}{(u+s)^{j+1}} d u \\
& =O\left(\frac{1}{|s|^{j-1}}\right)
\end{aligned}
$$


for $j \in \mathbb{Z}_{\geq 2}$. Hence the approximate formula for $\left(\Gamma^{(r)} / \Gamma\right)(s)$ is

$$
\begin{aligned}
\frac{\Gamma^{(r)}}{\Gamma}(s) & =\left(G^{(1)}(s)\right)^{r}+O\left(\sum_{1 q_{1}+\cdots+r q_{r}=r, q_{1} \neq r} \prod_{j=1}^{r}\left|G^{(j)}(s)\right|^{q_{j}}\right) \\
= & \left(\log s+O\left(\frac{1}{|s|}\right)\right)^{r}+O\left(\frac{|\log s|^{r-1}}{|s|}\right)=(\log s)^{r} \sum_{j=0}^{r} \frac{M_{j}(s)}{(\log s)^{j}}
\end{aligned}
$$

for $s \in D$ and $r \in \mathbb{Z}_{\geq 0}$, where $M_{j}(s)=O\left(1 /|s|^{j}\right)$ for $j \in \mathbb{Z}_{\geq 1}$ and $M_{0}(s)=1$. Using (2.7) and the approximate formula

(2.8) $\log \left(s \pm \frac{k-1}{2}\right)=\log s+\log \left(1 \pm \frac{k-1}{2 s}\right)=\log |s|+i \arg s+O(1 /|s|)$ for $|s|>(k-1) / 2$, we can write

$$
\begin{aligned}
\left.\frac{\left(\Gamma\left(s-\frac{k-1}{2}\right)\right.}{\Gamma\left(s-\frac{k-1}{2}\right)} \Gamma\left(s+\frac{k-1}{2}\right)\right)^{(l)} \\
=\sum_{j=0}^{l}\left(\begin{array}{l}
l \\
j
\end{array}\right) \frac{\Gamma^{(j)}}{\Gamma}\left(s-\frac{k-1}{2}\right) \frac{\Gamma^{(l-j)}}{\Gamma}\left(s+\frac{k-1}{2}\right) \\
=\sum_{j=0}^{l}\left(\begin{array}{l}
l \\
j
\end{array}\right)\left(\log \left(s-\frac{k-1}{2}\right)\right)^{j}\left(\log \left(s+\frac{k-1}{2}\right)\right)^{l-j} \\
\times \sum_{\substack{0 \leq j_{1}+j_{2} \leq l \\
0 \leq j_{1} \leq j, 0 \leq j_{2} \leq l-j}} \frac{M_{j_{1}}\left(s-\frac{k-1}{2}\right)}{\left(\log \left(s-\frac{k-1}{2}\right)\right)^{j_{1}}} \frac{M_{j_{2}}\left(s+\frac{k-1}{2}\right)}{\left(\log \left(s+\frac{k-1}{2}\right)\right)^{j_{2}}} \\
=S_{l}(s)+T_{l}(s)
\end{aligned}
$$

for $l \in \mathbb{Z}_{\geq 0}, s \in D^{\prime}$ and $|s|>(k-1) / 2$, where $D^{\prime}:=\mathbb{C} \backslash\{s \in \mathbb{C} \mid \operatorname{Re} s<$ $(k-1) / 2+\varepsilon,|\operatorname{Im} s|<\varepsilon\}$ and

$$
S_{l}(s)=\left(\log \left(s-\frac{k-1}{2}\right)+\log \left(s+\frac{k-1}{2}\right)\right)^{l},
$$

$$
T_{l}(s)=O\left(\frac{1}{|s| \log |s|} \sum_{j=0}^{l}(\log |s|)^{j}(\log |s|)^{l-j}\right)=O\left(\frac{(\log |s|)^{l-1}}{|s|}\right)
$$

for $l \in \mathbb{Z}_{\geq 1}$, in particular $S_{0}(s)=1$ and $T_{0}(s)=0$.

Next using $R_{r}(s), S_{r}(s)$ and $T_{r}(s)$, we shall give a condition which implies $|f(s)|>|g(s)|$ for some region. From (2.5) and (2.9), the inequality $|f(s)|>$ $|g(s)|$ is equivalent to

$$
\left|S_{m}(s)+T_{m}(s)\right|>\left|\sum_{r=0}^{m-1} \frac{R_{r}}{R_{0}}(s)\left(S_{r}(s)+T_{r}(s)\right)\right| .
$$


Dividing both sides by $S_{m-1}(s)$ and applying the triangle inequality, we see that if

$$
\left|S_{1}(s)\right|>\left|\frac{T_{m}}{S_{m-1}}(s)\right|+\left|\sum_{r=0}^{m-1} \frac{R_{r}}{R_{0}}(s)\left(\frac{1}{S_{m-1-r}}(s)+\frac{T_{r}}{S_{m-1}}(s)\right)\right|,
$$

then $|f(s)|>|g(s)|$ for $s \in D^{\prime}$ and $|s|>(k-1) / 2$. To prove (2.11), we shall obtain upper bounds of $\left(1 / S_{r}\right)(s)$ and $\left(R_{r} / R_{0}\right)(s)$. The formula 2.8) gives

$$
\left|\frac{1}{S_{r}}(s)\right| \leq \frac{C_{1}}{(\log |s|)^{r}}
$$

for the above $s$ and $r \in \mathbb{Z}_{\geq 0}$; here and later, $C_{1}, C_{2}, \ldots$ denote positive constants depending on $f, r$ and $\varepsilon$. Since $L_{f}^{(j)}(s)$ and $\left(1 / L_{f}\right)(s)$ are absolutely convergent for $\operatorname{Re} s>1$, it follows that

$$
\left|\frac{R_{r}}{R_{0}}(s)\right|=\left|\sum_{j=0}^{r} a_{j}^{\prime} \frac{L_{f}^{(j)}}{L_{f}}(s)+\tan (\pi s) \sum_{j=0}^{r} b_{j}^{\prime} \frac{L_{f}^{(j)}}{L_{f}}(s)\right| \leq C_{2}+C_{3}|\tan (\pi s)|
$$

for $\operatorname{Re} s \geq 1+\varepsilon$. Here $\tan (\pi s)$ is estimated as

$$
|\tan \pi(\sigma+i t)|=\left|\frac{e^{-2 t} e^{2 \pi i \sigma}-1}{e^{-2 t} e^{2 \pi i \sigma}+1}\right| \leq \begin{cases}2 /\left(1-e^{-2 \varepsilon}\right) & \text { if }|t| \geq \varepsilon, \\ 3 & \text { if } \sigma \in \mathbb{Z} .\end{cases}
$$

Combining 2.10 and 2.12 -2.14), we see that the right-hand side of (2.11) is estimated as

$$
\begin{aligned}
& \left|\frac{T_{m}}{S_{m-1}}(s)\right|+\left|\sum_{r=0}^{m-1} \frac{R_{r}}{R_{0}}(s)\left(\frac{1}{S_{m-1-r}}(s)+\frac{T_{r}}{S_{m-1}}(s)\right)\right| \\
\leq & \frac{C_{4}}{|s|}+C_{5}|\tan (\pi s)| \sum_{r=0}^{m-1}\left(\frac{1}{(\log |s|)^{m-1-r}}+\frac{1}{|s|(\log |s|)^{m-r}}\right) \leq C_{f, m, \varepsilon}
\end{aligned}
$$

for $|s|>(k-1) / 2$ and $\operatorname{Re} s \geq 1+\varepsilon$ provided $|\operatorname{Im} s| \geq \varepsilon$ or $\operatorname{Re} s \in \mathbb{Z}$, where $C_{f, m, \varepsilon}$ is a positive constant depending on $f, m$ and $\varepsilon$. Choose $r_{f, m, \varepsilon} \in$ $\mathbb{R}_{>(k-1) / 2}$ such that $C_{f, m, \varepsilon}<\left(\log r_{f, m, \varepsilon}\right) / C_{1}$. The inequalities 2.12) and 2.15) imply that 2.11 is true, that is, $L_{f}^{(m)}(1-s)$ has no zero for $s \in \mathbb{C}$ such that $|s| \geq r_{f, m, \varepsilon}, \operatorname{Re} s \geq 1+\varepsilon$ and $|\operatorname{Im} s| \geq \varepsilon$. Therefore, we conclude that for any $\varepsilon \in \mathbb{R}_{>0}$ there exists $\delta_{f, m, \varepsilon} \in \mathbb{R}_{>(k-1) / 2+1}$ such that $L_{f}^{(m)}(s)$ has no zero with $|s| \geq \delta_{f, m, \varepsilon}$, Re $s \leq-\varepsilon$ and $|\operatorname{Im} s| \geq \varepsilon$, that is, the proof of (ii) is complete.

Finally, we show (iii) by applying Rouché's theorem to $f(s)$ and $g(s)$. For $n \in \mathbb{Z}_{\geq 1}$ let $D_{n}$ be the region where $n \leq \operatorname{Re} s \leq n+1$ and $|\operatorname{Im} s| \leq 1 / 2$. By (2.14) and 2.15), we see that there exists $\delta_{f, m, 1 / 2} \in \mathbb{R}_{>(k-1) / 2}$ such that $|f(s)|>|g(s)|$ on the boundary of $D_{n}$ and in the region where $|s|>\delta_{f, m, 1 / 2}$ and $\operatorname{Re} s \geq 1+1 / 2$. Then the number of zeros of $f(s)$ is equal to that of 
$f(s)+g(s)$ in the interior of $D_{n}$. From 2.4, 2.5 and 2.9),

$$
f(s)=L_{f}(s) \cos (\pi s) \Gamma\left(s-\frac{k-1}{2}\right) \Gamma\left(s+\frac{k-1}{2}\right)\left(S_{m}(s)+T_{m}(s)\right) .
$$

When $R_{f, m}$ is chosen such that $R_{f, m} \geq \delta_{f, m, 1 / 2}$ and $C_{6} /\left(R_{f, m} \log R_{f, m}\right)<1$, 2.12 gives

$$
\left|\frac{T_{m}}{S_{m}}(s)\right| \leq \frac{C_{6}}{|s| \log |s|}<1,
$$

that is, $S_{m}(s)+T_{m}(s)$ has no zero with $|s| \geq R_{f, m}$. Hence $f(s)$ has the only real zero $s=n+1 / 2$ in $D_{n}$. It is clear that $\overline{f(s)}=f(\bar{s})$ and $\overline{g(s)}=g(\bar{s})$ for $s \in \mathbb{C}$, which implies that $L_{f}^{(m)}(1-s)$ can only have a real zero in the interior of $D_{n}$. Replacing $1-s$ with $s$, we conclude that there exists $\alpha_{f, m} \in \mathbb{R}_{<-(k-1) / 2-1}$ such that $L_{f}^{(m)}(s)$ has no complex zero in $\operatorname{Re} s<\alpha_{f, m}$ and one real zero in each open interval $(n-1, n)$ for $n \in \mathbb{Z}_{\leq \alpha_{f, m}}$. The proof of (iii) is complete.

3. Proof of Theorem 1.2. Using Theorem 1.1, we can choose $\alpha_{f, m} \in$ $\mathbb{R}_{<-(k-1) / 2}$ and $\sigma_{f, m} \in \mathbb{R}_{>1}$ such that $L_{f}^{(m)}(s)$ has no zeros with $\operatorname{Re} s \leq \alpha_{f, m}$ or Re $s \geq \sigma_{f, m}$. Moreover, choose $\tau_{f, m} \in \mathbb{R}_{>2}$ and $T \in \mathbb{R}_{>0}$ such that $L_{f}^{(m)}(s)$ has no zeros with $0<\operatorname{Im} s \leq \tau_{f, m}$ or $\operatorname{Im} s=T$. Using the residue theorem in the region where $\alpha_{f, m} \leq \operatorname{Re} s \leq \sigma_{f, m}$ and $\tau_{f, m} \leq \operatorname{Im} s \leq T$, we get

$$
\begin{aligned}
N_{f, m}(T)=\frac{1}{2 \pi i}( & \int_{\alpha_{f, m}+i \tau_{f, m}}^{\sigma_{f, m}+i \tau_{f, m}}+\int_{\sigma_{f, m}+i \tau_{f, m}}^{\sigma_{f, m}+i T}+\int_{\sigma_{f, m}+i T}^{\alpha_{f, m}+i T} \\
& \left.+\int_{\alpha_{f, m}+i T}^{\alpha_{f, m}+i \tau_{f, m}}\right)\left(\log L_{f}^{(m)}(s)\right)^{\prime} d s=: I_{1}+I_{2}+I_{3}+I_{4} .
\end{aligned}
$$

First, it is clear that

$$
I_{1}=\frac{\log L_{f}^{(m)}\left(\sigma_{f, m}+i \tau_{f, m}\right)-\log L_{f}^{(m)}\left(\alpha_{f, m}+i \tau_{f, m}\right)}{2 \pi i}=O(1) .
$$

To approximate $I_{2}$, we write $L_{f}^{(m)}(s)=\lambda_{f}\left(n_{f}\right)\left(-\log n_{f}\right)^{m} F(s) n_{f}^{-s}$ where $F(s)$ is given by (2.1). Using (2.2) we find that $1 / 2 \leq|F(s)| \leq 3 / 2$, $\operatorname{Re} F(s) \geq 1 / 2$ and $|\arg F(s)|<\pi / 2$ for $s=\sigma_{f, m}+i t(t \in \mathbb{R})$. Hence

$$
\begin{aligned}
I_{2} & =\frac{1}{2 \pi i}\left[\log \frac{\lambda_{f}\left(n_{f}\right)\left(-\log n_{f}\right)^{m}}{n_{f}^{s}}+\log F(s)\right]_{\sigma_{f, m}+i \tau_{f, m}}^{\sigma_{f, m}+i T} \\
& =\frac{-\left(\sigma_{f, m}+i T\right) \log n_{f}}{2 \pi i}+O(1)=-\frac{T}{2 \pi} \log n_{f}+O(1) .
\end{aligned}
$$


Next we shall estimate $I_{3}$. By (1.8), the approximate functional equation for $L_{f}^{(m)}(s)$ (see [15, Theorem 1.2]) and Rankin's result, there exists $A \in \mathbb{R}_{\geq 0}$ such that $L_{f}^{(m)}(\sigma+i t)=O\left(|t|^{A}\right)$ uniformly for $\sigma \in\left[\alpha_{f, m}, \sigma_{f, m}\right]$. This implies that

$$
\begin{aligned}
I_{3} & =\frac{\log L_{f}^{(m)}\left(\alpha_{f, m}+i T\right)-\log L_{f}^{(m)}\left(\sigma_{f, m}+i T\right)}{2 \pi i} \\
& =\frac{\arg L_{f}^{(m)}\left(\alpha_{f, m}+i T\right)-\arg L_{f}^{(m)}\left(\sigma_{f, m}+i T\right)}{2 \pi}+O(\log T) .
\end{aligned}
$$

To estimate the first term of the right-hand side, we write $L_{f}^{(m)}(\sigma+i T)=$ $(-1)^{m} e^{-i T \log n_{f}} \lambda_{f}\left(n_{f}\right) G(\sigma+i T)$ where

$$
G(\sigma+i T)=\frac{\left(\log n_{f}\right)^{m}}{n_{f}^{\sigma}}+\frac{1}{\lambda_{f}\left(n_{f}\right)} \sum_{n=n_{f}+1}^{\infty} \frac{\lambda_{f}(n)(\log n)^{m}}{n^{\sigma}} e^{i T \log n_{f} / n}
$$

for $\sigma \in \mathbb{R}_{>1}$. Let $Q$ be the number of zeros of $\operatorname{Re} G(s)$ on the line segment $\left(\alpha_{f, m}+i T, \sigma_{f, m}+i T\right)$. Divide this line into $Q+1$ subintervals by these zeros. Then on each subinterval, the sign of $\operatorname{Re} G(s)$ is constant, and the variation of $\arg G(s)$ is at most $\pi$. Hence, there exists a constant $C$ such that $\arg G(s)=\arg L_{f}^{(m)}(s)+C$ on the divided line, and so

$$
\left|\arg L_{f}^{(m)}\left(\alpha_{f, m}+i T\right)-\arg L_{f}^{(m)}\left(\sigma_{f, m}+i T\right)\right| \leq(Q+1) \pi .
$$

In order to estimate $Q$, let $H(z)=(G(z+i T)+\overline{G(\bar{z}+i T)}) / 2$. Then we find that

$$
\begin{aligned}
& H(\sigma)=\operatorname{Re} G(\sigma+i T) \\
& =\frac{\left(\log n_{f}\right)^{m}}{n_{f}^{\sigma}}\left(1+\sum_{n=n_{f}+1}^{\infty} \frac{\lambda_{f}(n)}{\lambda_{f}\left(n_{f}\right)}\left(\frac{\log n}{\log n_{f}}\right)^{m}\left(\frac{n_{f}}{n}\right)^{\sigma} \cos \left(T \log \frac{n_{f}}{n}\right)\right)
\end{aligned}
$$

for $\sigma \in \mathbb{R}_{>1}$. Now (2.2) and (3.6) give

$$
\frac{1}{2} \frac{\left(\log n_{f}\right)^{m}}{n_{f} \sigma_{f, m}} \leq H\left(\sigma_{f, m}\right) \leq \frac{3}{2} \frac{\left(\log n_{f}\right)^{m}}{n_{f} \sigma_{f, m}} .
$$

Take $T$ so large that $T-\tau_{f, m}>2\left(\sigma_{f, m}-\alpha_{f, m}\right)$. Since $\operatorname{Im}(z+i T) \geq$ $T-\left(T-\tau_{f, m}\right)>0$ for $z \in \mathbb{C}$ such that $\left|z-\sigma_{f, m}\right|<T-\tau_{f, m}$, it follows that $H(z)$ is analytic in the disc $\left|z-\sigma_{f, m}\right|<T-\tau_{f, m}$. Note that there exists a positive constant $B$ such that $H(z)=O\left(T^{B}\right)$ in that disc because $L_{f}(\sigma+i t)$ $=O\left(|t|^{A}\right)$. For $u \in \mathbb{R}_{\geq 0}$, let $P(u)$ be the number of zeros of $H(z)$ in $\left|z-\sigma_{f, m}\right| \leq u$. Then using the trivial estimate 


$$
P\left(\sigma_{f, m}-\alpha_{f, m}\right) \leq \frac{1}{\log 2} \int_{\sigma_{f, m}-\alpha_{f, m}}^{2\left(\sigma_{f, m}-\alpha_{f, m}\right)} \frac{P(u)}{u} d u \leq \frac{1}{\log 2} \int_{0}^{2\left(\sigma_{f, m}-\alpha_{f, m}\right)} \frac{P(u)}{u} d u,
$$

Jensen's formula (see [13, Chapter 3.61]), the above remark and (3.7), we have

$$
\begin{aligned}
P\left(\sigma_{f, m}-\alpha_{f, m}\right) & \ll \int_{0}^{2\left(\sigma_{f, m}-\alpha_{f, m}\right)} \frac{P(u)}{u} d u \\
& =\frac{1}{2 \pi} \int_{0}^{2 \pi} \log \left|H\left(\sigma_{f, m}+2\left(\sigma_{f, m}-\alpha_{f, m}\right) e^{i \theta}\right)\right| d \theta-\log \left|H\left(\sigma_{f, m}\right)\right| \\
& \ll \int_{0}^{2 \pi} \log T^{B} d \theta+1 \ll \log T
\end{aligned}
$$

Therefore

$$
Q=\#\left\{\sigma \in\left(\alpha_{f, m}, \sigma_{f, m}\right) \mid F(\sigma)=0\right\} \ll P\left(\sigma_{f, m}-\alpha_{f, m}\right) \ll \log T .
$$

Combining (3.4), (3.5), (3.8), we obtain

$$
I_{3}=O(\log T) .
$$

Finally, in order to approximate $I_{4}$, we shall obtain an approximate formula for $\log L_{f}^{(m)}\left(\alpha_{f, m}+i T\right)$ as $T \rightarrow \infty$. By the proof of Theorem 1.1, there exists $\delta_{f, m} \in \mathbb{R}_{>0}$ such that

$$
\left|\frac{g}{f}(1-s)\right|<1, \quad\left|\frac{T_{m}}{S_{m}}(1-s)\right|<1
$$

for $s \in \mathbb{C}$ in the region where $|s-(1-(k-1) / 2)|>\delta_{f, m}, \operatorname{Re} s<1-(k-1) / 2$ and $|\operatorname{Im} s|>1 / 2$. Choose $\alpha_{f, m} \in \mathbb{R}_{<0}$ such that $\alpha_{f, m}<1-(k-1) / 2-\delta_{f, m}$. Then the path of $I_{4}$ is contained in the above region. Replacing $s$ with $1-s$ and taking the logarithm of both sides of 2.5 , we obtain

$$
\begin{aligned}
\log L_{f}^{(m)}\left(\alpha_{f, m}\right. & +i T) \\
= & -2\left(1-\alpha_{f, m}-i T\right) \log 2 \pi+\log f\left(1-\alpha_{f, m}-i T\right) \\
& +\log \left(1+\frac{g}{f}\left(1-\alpha_{f, m}-i T\right)\right)+O(1) .
\end{aligned}
$$

The first inequality of 3.10$)$ gives $\left|\arg \left(1+(g / f)\left(1-\alpha_{f, m}-i T\right)\right)\right|<\pi / 2$ and

$$
\begin{aligned}
& \log \left(1+\frac{g}{f}\left(1-\alpha_{f, m}-i T\right)\right) \\
& \ll \sqrt{\left|1+\frac{g}{f}\left(1-\alpha_{f, m}-i T\right)\right|^{2}+\left(\arg \left(1+\frac{g}{f}\left(1-\alpha_{f, m}-i T\right)\right)\right)^{2}} \ll 1 .
\end{aligned}
$$


By (2.16), the second term of the right-hand side of (3.11) can be written as

$$
\begin{aligned}
& \log f\left(1-\alpha_{f, m}-i T\right) \\
= & \log \Gamma\left(1-\alpha_{f, m}-\frac{k-1}{2}-i T\right)+\log \Gamma\left(1-\alpha_{f, m}+\frac{k-1}{2}-i T\right) \\
& +\log S_{m}\left(1-\alpha_{f, m}-i T\right)+\log \left(1+\frac{T_{m}}{S_{m}}\left(1-\alpha_{f, m}-i T\right)\right) \\
& +\log L_{f}\left(1-\alpha_{f, m}-i T\right)+\log \cos \pi\left(1-\alpha_{f, m}-i T\right) .
\end{aligned}
$$

Now it is clear that

$$
\begin{aligned}
& \cos \left(\pi\left(1-\alpha_{f, m}-i T\right)\right)=e^{\pi T} e^{i\left(1-\alpha_{f, m}\right)-\log 2}\left(1+e^{-2 \pi\left(1-\alpha_{f, m}\right) i} / e^{2 \pi T}\right), \\
& \log L_{f}\left(1-\alpha_{f, m}-i T\right)=\sum_{n=1}^{\infty} \frac{b_{f}(n)}{n^{1-\alpha_{f, m}-i T}}
\end{aligned}
$$

where $b_{f}(n)$ is given by

$$
b_{f}(n)= \begin{cases}\left(\alpha_{f}(p)^{r}+\beta_{f}(p)^{r}\right) / r, & n=p^{r}, \\ 0, & \text { otherwise, }\end{cases}
$$

and $\alpha_{f}(p), \beta_{f}(p)$ are given by (1.1). Hence for the last two terms of the right-hand side of (3.13) we have

$$
\log L_{f}\left(1-\alpha_{f, m}-i T\right)+\log \cos \left(\pi\left(1-\alpha_{f, m}-i T\right)\right)=\pi T+O(1) .
$$

By a similar discussion to (3.12), the fourth term of the right-hand side of 3.13 is estimated as

$$
\log \left(1+\frac{T_{m}}{S_{m}}\left(1-\alpha_{f, m}-i T\right)\right) \ll 1 .
$$

The trivial formula

$$
\log \left(1-\alpha_{f, m} \pm \frac{k-1}{2}-i T\right)=\log T-(\pi / 2) i+O(1 / T)
$$

shows that the third term of the right-hand side of $(3.13)$ is approximated as

(3.16) $\quad \log S_{m}\left(1-\alpha_{f, m}-i T\right)$

$$
\begin{aligned}
& =m \log \left(\log \left(1-\alpha_{f, m}-\frac{k-1}{2}-i T\right)+\log \left(1-\alpha_{f, m}+\frac{k-1}{2}-i T\right)\right) \\
& =m \log \log T+O(1) .
\end{aligned}
$$

Using Stirling's formula

$$
\log \Gamma(s)=(s-1 / 2) \log s-s+\log \sqrt{2 \pi}+O(1 /|s|)
$$


and the approximate formula for $\log \left(1-\alpha_{f, m} \pm(k-1) / 2-i T\right)$, we approximate the sum of the first two terms of the right-hand side of (3.13) as

$$
\begin{aligned}
& \text { 3.17) } \quad \log \Gamma\left(1-\alpha_{f, m}+\frac{k-1}{2}-i T\right)+\log \Gamma\left(1-\alpha_{f, m}-\frac{k-1}{2}-i T\right) \\
& =\left(1-2 \alpha_{f, m}-2 i T\right)(\log T-(\pi / 2) i+O(1 / T))-2\left(1-\alpha_{f, m}-i T\right)+O(1) \\
& =-2 i T \log (T / e)-\pi T+\left(1-2 \alpha_{f, m}\right) \log T+O(1) .
\end{aligned}
$$

Combining (3.11)-(3.17), we obtain the desired approximate formula

$$
\log L_{f}^{(m)}\left(\alpha_{f, m}+i T\right)=-2 i T \log \frac{T}{2 \pi e}+O(\log T),
$$

which implies that

$$
I_{4}=\frac{T}{\pi} \log \frac{T}{2 \pi e}+O(\log T) .
$$

By (3.2), 3.3), 3.9) and 3.18), the proof of Theorem 1.2 is complete.

4. Proof of Theorem 1.3. Write $L_{f}^{(m)}(s)=\lambda_{f}\left(n_{f}\right)\left(-\log n_{f}\right)^{m} F(s) / n_{f}^{s}$ where $F(s)$ is given by (2.1). By the proof of Theorem 1.1, we can choose $\sigma_{f, m} \in \mathbb{R}_{>1}$ such that $L_{f}(s)$ has no zero with $\operatorname{Re} s>\sigma_{f, m}$, and

$$
\sum_{n=n_{f}+1}^{\infty}\left|\frac{\lambda_{f}(n)}{\lambda_{f}\left(n_{f}\right)}\right|\left(\frac{\log n}{\log n_{f}}\right)^{m}\left(\frac{n_{f}}{n}\right)^{\sigma_{f, m} / 2} \leq \frac{1}{2} .
$$

Note that $(2.2)$ and the above inequality give

$$
\begin{aligned}
|F(s)-1| & \leq \sum_{n=n_{f}+1}^{\infty}\left|\frac{\lambda_{f}(n)}{\lambda_{f}\left(n_{f}\right)}\right|\left(\frac{\log n}{\log n_{f}}\right)^{m}\left(\frac{n_{f}}{n}\right)^{\sigma_{f, m} / 2+\sigma / 2} \\
& \leq \frac{1}{2}\left(\frac{n_{f}}{n_{f}+1}\right)^{\sigma / 2}
\end{aligned}
$$

for $\operatorname{Re} s \geq \sigma_{f, m}$. Applying Littlewood's formula (see [13, Chapter 3.8]) to $F(s)$, we obtain

$$
\begin{aligned}
& 2 \pi \sum_{\substack{F(\rho)=0 \\
\sigma \leq \operatorname{Re} \rho \leq \sigma, m \\
1 \leq \operatorname{Im} \rho \leq T}}(\operatorname{Re} \rho-\sigma) \\
&= \int_{1}^{T} \log |F(\sigma+i t)| d t-\int_{1}^{T} \log \left|F\left(\sigma_{f, m}+i t\right)\right| d t \\
&+\int_{\sigma}^{\sigma_{f, m}} \arg F(u+i T) d t-\int_{\sigma}^{\sigma_{f, m}} \arg F(u+i) d t \\
&=: I_{1}+I_{2}+I_{3}+I_{4}
\end{aligned}
$$


for $\sigma \in \mathbb{R}_{>1 / 2}$. We first estimate $I_{2}$. Cauchy's theorem gives

$$
I_{2}=\int_{1}^{T} \log |F(v+i t)| d t+\int_{\sigma_{f, m}}^{v} \log |F(u+i)| d u-\int_{\sigma_{f, m}}^{v} \log |F(u+i T)| d u
$$

for all $v>\sigma_{f, m}$. The facts that $\log |X| \leq|X-1|$ for $X \in \mathbb{C}$ and $-\log |Y| \leq$ $2|Y-1|$ for $Y \in \mathbb{C}$ satisfying $|Y| \geq 1 / 2$, and (4.1), imply that

$$
\int_{1}^{T} \log |F(v+i t)| d t \leq \frac{T-1}{2}\left(\frac{n_{f}}{n_{f}+1}\right)^{v / 2}
$$

and

$$
\begin{aligned}
\int_{\sigma_{f, m}}^{v} \log |F(u+i)| d u- & \int_{\sigma_{f, m}}^{v} \log \mid F(u \\
& +i T) \mid d u \\
& \ll \int_{\sigma_{f, m}}^{v}\left(\frac{n_{f}}{n_{f}+1}\right)^{u / 2} d u \ll 1 .
\end{aligned}
$$

Combining (4.3)- 4.5 we get

$$
I_{2}=O(1)
$$

Following the estimation of $I_{3}$ in the proof of Theorem 1.2 , we obtain

$$
I_{3}+I_{4}=O(\log T) .
$$

To estimate $I_{1}$, we calculate

$$
I_{1}=\frac{T-1}{2} \log \frac{n_{f}^{2 \sigma}}{\left|\lambda_{f}\left(n_{f}\right)\right|^{2}\left(\log n_{f}\right)^{2 m}}+\frac{1}{2} \int_{1}^{T} \log \left|L_{f}^{(m)}(\sigma+i t)\right|^{2} d t .
$$

Jensen's inequality gives

$$
\int_{1}^{T} \log \left|L_{f}^{(m)}(\sigma+i t)\right|^{2} d t \leq(T-1) \log \left(\frac{1}{T-1} \int_{1}^{T}\left|L_{f}^{(m)}(\sigma+i t)\right|^{2} d t\right) .
$$

Combining (4.2) and (4.6)-4.9), we obtain

$$
\begin{aligned}
\sum_{\substack{F(\rho)=0 \\
\sigma \leq \operatorname{Re} \rho \leq \sigma, m \\
1 \leq \operatorname{Im} \rho \leq T}}(\operatorname{Re} \rho-\sigma) \leq & \frac{T-1}{4 \pi} \log \left(\frac{1}{T-1} \int_{1}^{T}\left|L_{f}^{(m)}(\sigma+i t)\right|^{2} d t\right) \\
& +\frac{T-1}{4 \pi} \log \frac{n_{f}^{2 \sigma}}{\left|\lambda_{f}\left(n_{f}\right)\right|^{2}\left(\log n_{f}\right)^{2 m}}+O(\log T) .
\end{aligned}
$$

First, we consider the mean square of $L_{f}^{(m)}(s)$ for $\operatorname{Re} s>1$. We calculate as follows: 


$$
\begin{aligned}
& \text { (4.11) } \quad \int_{1}^{T}\left|L_{f}^{(m)}(\sigma+i t)\right|^{2} d t \\
& =\sum_{n_{1}, n_{2}=1}^{\infty} \frac{\overline{\lambda_{f}\left(n_{1}\right)} \lambda_{f}\left(n_{2}\right)\left(\log n_{1}\right)^{m}\left(\log n_{2}\right)^{m}}{\left(n_{1} n_{2}\right)^{\sigma}} \int_{\max \left\{n_{1}, n_{2}\right\}}^{T}\left(\frac{n_{1}}{n_{2}}\right)^{i t} d t \\
& =(T-1) \sum_{n=1}^{\infty} \frac{\left|\lambda_{f}(n)\right|^{2}(\log n)^{2 m}}{n^{2 \sigma}} \\
& +\frac{1}{i} \sum_{\substack{n_{1}, n_{2}=1 \\
n_{1} \neq n_{2}}}^{\infty} \frac{\overline{\lambda_{f}\left(n_{1}\right) n_{1}^{-i T}} \lambda_{f}\left(n_{2}\right) n_{2}^{-i T}\left(\log n_{1}\right)^{m}\left(\log n_{2}\right)^{m}}{\left(n_{1} n_{2}\right)^{\sigma} \log \left(n_{1} / n_{2}\right)} \\
& -\frac{1}{i} \sum_{\substack{n_{1}, n_{2}=1 \\
n_{1} \neq n_{2}}}^{\infty} \frac{\overline{\lambda_{f}\left(n_{1}\right) n_{1}^{-i \max \left\{n_{1}, n_{2}\right\}}} \lambda_{f}\left(n_{2}\right) n_{2}^{-i \max \left\{n_{1}, n_{2}\right\}}\left(\log n_{1}\right)^{m}\left(\log n_{2}\right)^{m}}{\left(n_{1} n_{2}\right)^{\sigma} \log \left(n_{1} / n_{2}\right)} .
\end{aligned}
$$

By the same discussion for $U_{\sigma}(x)$ with

$$
\begin{aligned}
\left(\alpha_{n_{1}}, \beta_{n_{2}}\right)= & \left(\lambda_{f}\left(n_{1}\right) n_{1}^{-i T}\left(\log n_{1}\right)^{m}, \lambda_{f}\left(n_{2}\right) n_{2}^{-i T}\left(\log n_{2}\right)^{m}\right) \quad \text { or } \\
& \left(\lambda_{f}\left(n_{1}\right) n_{1}^{-i \max \left\{n_{1}, n_{2}\right\}}\left(\log n_{1}\right)^{m}, \lambda_{f}\left(n_{2}\right) n_{2}^{-i \max \left\{n_{1}, n_{2}\right\}}\left(\log n_{2}\right)^{m}\right)
\end{aligned}
$$

in [3, p. 348, Lemma 6], we find that the second and third terms on the right-hand side of (4.11) are $O(1)$ uniformly for $\sigma>1$. Hence, for $\sigma>1$,

$$
\int_{1}^{T}\left|L_{f}^{(m)}(\sigma+i t)\right|^{2} d t=(T-1) \sum_{n=1}^{\infty} \frac{\left|\lambda_{f}(n)\right|^{2}(\log n)^{2 m}}{n^{2 \sigma}}+O(1) .
$$

Next the mean square of $L_{f}^{(m)}(s)$ for $1 / 2<\operatorname{Re} s \leq 1$ is obtained as follows:

Lemma 4.1 ([15, Theorem 1.3]). For any $m \in \mathbb{Z}_{\geq 0}$ and $T>0$, we have

$$
\int_{1}^{T}\left|L_{f}^{(m)}(\sigma+i t)\right|^{2} d t
$$

$$
= \begin{cases}(T-1) \sum_{n=1}^{\infty} \frac{\left|\lambda_{f}(n)\right|^{2}(\log n)^{2 m}}{n^{2 \sigma}}+O\left(T^{2(1-\sigma)}(\log T)^{2 m}\right), & 1 / 2<\sigma<1, \\ (T-1) \sum_{n=1}^{\infty} \frac{\left|\lambda_{f}(n)\right|^{2}(\log n)^{2 m}}{n^{2 \sigma}}+O\left((\log T)^{2 m+2}\right), & \sigma=1 .\end{cases}
$$

Using Rankin's result mentioned in Introduction and the fact that

$$
\int_{n_{f}}^{\infty} \frac{(\log u)^{2 m}}{u^{2 \sigma}} d u=\frac{(2 m) ! n_{f}^{1-2 \sigma}}{(2 \sigma-1)^{2 m+1}} \sum_{j=0}^{2 m} \frac{\left(\log n_{f}\right)^{j}(2 \sigma-1)^{j}}{j !},
$$


which is obtained by induction and integration by parts, we find that the series in the main term of (4.13) is approximated as

$$
\begin{aligned}
\sum_{n=1}^{\infty} & \frac{\left|\lambda_{f}(n)\right|^{2}(\log n)^{2 m}}{n^{2 \sigma}} \\
& =-\int_{n_{f}}^{\infty}\left(\frac{(\log u)^{2 m}}{u^{2 \sigma}}\right)^{\prime} \sum_{n_{f}<n \leq u}\left|\lambda_{f}(n)\right|^{2} d u \\
& =-\frac{C_{f}\left(\log n_{f}\right)^{2 m}}{n_{f}^{2 \sigma-1}}+C_{f} \int_{n_{f}}^{\infty} \frac{(\log u)^{2 m}}{u^{2 \sigma}} d u+O\left(\int_{n_{f}}^{\infty} \frac{(\log u)^{2 m}}{u^{2 \sigma+2 / 5}} d u\right) \\
& =\frac{(2 m) ! n_{f} C_{f}}{n_{f}^{2 \sigma}} \frac{1}{(2 \sigma-1)^{2 m+1}}+O\left(\frac{1}{(2 \sigma-1)^{2 m}}\right)
\end{aligned}
$$

as $\sigma \rightarrow 1 / 2+0$. From (4.10)-(4.14), the following approximate formula is obtained:

$$
\begin{aligned}
& \sum_{\begin{array}{c}
F(\rho)=0, \\
\sigma \leq \operatorname{Re} \rho \leq \sigma_{f, m} \\
1 \leq \operatorname{Im} \rho \leq T
\end{array}}(\operatorname{Re} \rho-\sigma) \\
& \leq \frac{(2 m+1)(T-1)}{4 \pi} \log \frac{1}{2 \sigma-1}+\frac{T-1}{4 \pi} \log \frac{(2 m) ! n_{f} C_{f}}{\left|\lambda_{f}\left(n_{f}\right)\right|^{2}\left(\log n_{f}\right)^{2 m}}+O(\log T) \\
& +\frac{T-1}{4 \pi} \begin{cases}\log \left(1+O\left(\frac{(2 \sigma-1)^{2 m+1}(\log T)^{2 m}}{T^{2 \sigma-1}}\right)\right), & 1 / 2<\sigma<1, \\
\log \left(1+O\left(\frac{(2 \sigma-1)^{2 m+1}(\log T)^{2 m+2}}{T}\right)\right), & \sigma=1, \\
\log \left(1+O\left(\frac{(2 \sigma-1)^{2 m+1}}{T}\right)\right), & \sigma>1 .\end{cases}
\end{aligned}
$$

Finally, we shall give an upper bound of $N_{f, m}(\sigma, T)$. Since $N_{f, m}(\sigma, T)$ is decreasing with respect to $\sigma$, it follows that

$$
\begin{aligned}
N_{f, m}(\sigma, T) & =N_{f, m}(\sigma, T)-N_{f, m}(\sigma, 1)+C \\
& \leq \frac{1}{\sigma-\sigma_{1}} \int_{\sigma_{1}}^{\sigma_{f, m}}\left(N_{f, m}(u, T)-N_{f, m}(u, 1)\right) d u+C,
\end{aligned}
$$

where we set $\sigma_{1}=1 / 2+(\sigma-1 / 2) / 2$. Note that $\sigma-\sigma_{1}=(\sigma-1 / 2) / 2$, so $2 \sigma_{1}-1=\sigma-1 / 2$. Since the number of zeros of $F_{m}(s)$ is equal to that of $L_{f}^{(m)}(s)$, it follows that 


$$
\begin{aligned}
& \sigma_{f, m} \\
& \int_{\sigma_{1}}\left(N_{f, m}(u, T)-N_{f, m}(u, 1)\right) d u \\
& =\int_{\sigma_{1}}^{\sigma_{f, m}} \sum_{\substack{F(\rho)=0 \\
u \leq \operatorname{Re} \rho \leq \sigma_{f, m} \\
1 \leq \operatorname{Im} \leq T}} 1 d u=\sum_{\substack{F(\rho)=0 \\
\sigma_{1} \leq \operatorname{Re} \rho \leq \sigma_{f, m} \\
1 \leq \operatorname{Im} \rho \leq T}} \int_{\sigma_{1}}^{\operatorname{Re} \rho} 1 d u \\
& =\sum_{\substack{F(\rho)=0 \\
\sigma_{1} \leq \operatorname{Re} \rho \leq \sigma_{f, m} \\
1 \leq \operatorname{Im} \rho \leq T}}\left(\operatorname{Re} \rho-\sigma_{1}\right) .
\end{aligned}
$$

Combining 4.15-4.17) we obtain (1.10) and (1.9). Hence the proof of Theorem 1.3 is complete.

\section{References}

[1] M. Aoki and M. Minamide, A zero density estimate for the derivatives of the Riemann zeta function, J. Algebra Number Theory Acad. 2 (2012), 361-375.

[2] B. C. Berndt, The number of zeros for $\zeta^{(k)}(s)$, J. London Math. Soc. (2) 2 (1970), $577-580$.

[3] A. Good, Approximative Funktionalgleichungen und Mittelwertsätze für Dirichletreihen, die Spitzenformen assoziiert sind, Comment. Math. Helv. 50 (1975), 327-361.

[4] E. Hecke, Über Modulfunktionen und die Dirichletschen Reihen mit Eulerscher Produktentwicklung. I, Math. Ann. 114 (1937), 1-28.

[5] A. A. Karatsuba and S. M. Voronin, The Riemann Zeta-Function, de Gruyter Expositions Math. 5, de Gruyter, 1992.

[6] C. G. Lekkerkerker, On the zeros of a class of Dirichlet series, Dissertation, Utrecht, 1955.

[7] N. Levinson and H. L. Montgomery, Zeros of the derivatives of the Riemann zetafunction, Acta Math. 133 (1974), 49-65.

[8] R. A. Rankin, Contributions to the theory of Ramanujan's function $\tau(n)$ and similar functions. II. The order of the Fourier coefficients of integral modular forms, Proc. Cambridge Philos. Soc. 35 (1939), 357-373.

[9] B. Riemann, Ueber die Anzahl der Primzahlen unter einer gegebenen Grösse, Monatsber. Königl. Preuss. Akad. Wiss. Berlin, 1859, 671-680.

[10] A. Speiser, Geometrisches zur Riemannschen Zetafunktion, Math. Ann. 110 (1934), $514-521$.

[11] R. Spira, Zero-free regions of $\zeta^{(k)}(s)$, J. London Math. Soc. 40 (1965), 677-682.

[12] R. Spira, Another zero-free region for $\zeta^{(k)}(s)$, Proc. Amer. Math. Soc. 26 (1970), 246-247.

[13] E. C. Titchmarsh, The Theory of Functions, Oxford Univ. Press, 1939.

[14] H. von Mangoldt, Zu Riemanns Abhandlung ,,Ueber die Anzahl der Primzahlen unter einer gegebenen Grösse“, J. Reine Angew. Math. 114 (1895), 255-305.

[15] Y. Yashiro, Approximate functional equation and mean value formula for the derivatives of L-functions attached to cusp forms, Funct. Approx. Comment. Math. 53 (2015), 97-122. 
[16] C. Y. Yıldirım, Zeros of $\zeta^{\prime \prime}(s) \& \zeta^{\prime \prime \prime}(s)$ in $\sigma<1 / 2$, Turk. J. Math. 24 (2000), 89-108.

Yoshikatsu Yashiro

Graduate School of Mathematics

Nagoya University

Nagoya 464-8602, Japan

E-mail: m09050b@math.nagoya-u.ac.jp 